\title{
Electroluminescence from bipolar resonant tunneling diodes
}

\author{
C. Van Hoof, J. Genoe, R. Mertens, and G. Borghs \\ Interuniversity Micro-Electronics Center (IMEC), Kapeldreef 75, B-3001 Leuven, Belgium \\ E. Goovaerts \\ Physics Department, University of Antwerp (U.I.A.), Universiteitsplein 1, B-2610 \\ Wilrijk-Antwerpen, Belgium
}

(Received 21 June 1991; accepted for publication 1 November 1991)

\begin{abstract}
The incorporation of an AIAs/GaAs resonant tunneling structure inside a GaAs $p$ - $n$ junction
leads to strong quantum-well electroluminescence originating from electron and hole tunneling into the resonant tunneling structure. A large peak-to-valley ratio of 10:1 in the electroluminescence intensity is achieved at the electron resonance at $4.2 \mathrm{~K}$, which decreases but persists $(1.45: 1)$ at room temperature. Resonant tunneling of holes is also apparent from the electroluminescence at low temperature.
\end{abstract}

Resonant tunneling of electrons in semiconductor heterostructures has bcen a subject of considerable interest because of its potential applications as ultra-high-speed electrical devices ${ }^{1,2}$ and multivalued logic switches. ${ }^{3,4}$ Although the resonant tunneling structure (RTS) seems suitable for optical modulation and detection purposes, ${ }^{5}$ only the optical detection has of yet been established. ${ }^{6}$

Photoluminescence (PL) characterization of operational RTS has considerably increased the understanding of charge buildup near and inside the quantum well of the RTS. ${ }^{7-14}$ It has been shown that the quantum-well PL under bias is rather electrophotoluminescence ${ }^{9}$ since it consists of the rccombination of majority-carrier electrons and minority-carrier photocreated holes that each tunnel from the opposite contact layer into the RTS. The bias dependence of the quantum-well PL intensity therefore reflects both the electron and the hole occupation in the quantum well. ${ }^{14}$ Large on-off ratios (15:1) in quantum-well PL have been observed between the on- and off-resonance conditions. Furthermore, in the electrical negative differential resistance (NDR) region, and due to internal resistance or external circuitry, the device can be oscillating, leading to fast optical oscillations of the PL intensity. Both the presence of such large on-off ratios in the light output of a device and the possibility of optical oscillations would be very desirable features in an electroluminescent device.

We have obtained such functions by growing a RTS inside the space-charge region of a $p-n$ junction. In this letter, we present the characteristics of this bipolar resonant tunneling light-emitting diode. This work contrasts with the observations of quantum-well electroluminescence by impact ionization ${ }^{15}$ in a unipolar RTS with a wide depletion layer that provides the required electric field resulting in the creation of holes.

The sample was grown by molecular-beam epitaxy (MBE) on a $p^{+}$-doped GaAs substrates and consisted of the following layers: $500-\mathrm{nm}$ GaAs $p^{+}\left(4 \times 10^{18} \mathrm{~cm}^{-3}\right)$ buffer layer, $50-\mathrm{nm}$ GaAs $p^{+}\left(4 \times 10^{17} \mathrm{~cm}^{-3}\right)$ spacer layer, 4-nm AlAs not intentionally doped (nid) barrier, 5.5-nm GaAs nid well, 4-nm AlAs nid barrier, 50-nm GaAs $n^{+}\left(4 \times 10^{17} \mathrm{~cm}^{-3}\right)$ spacer layer, and 300-nm GaAs $n^{+}\left(4 \times 10^{18} \mathrm{~cm}^{-3}\right)$ contact layer.

Standard lithography was used to define square mesas of $30 \times 30 \mu \mathrm{m}^{2}$. Fabrication involved a hollow-square $\mathrm{AuGe} / \mathrm{Ni} / \mathrm{Au}$ ohmic top contact stripe leaving optical windows available for $15 \times 15 \mu \mathrm{m}^{2}$. The ohmic contact was connected to a bonding pad on top of a silicon dioxide film that served for both isolation and passivation of the device mesas. The packaged sample was mounted in a continuoushelium-flow cryostat.

The current-voltage characteristics of the device in forward bias and beyond flat-band conditions at $4 \mathrm{~K}$ are shown in Fig. 1. Beyond the flat-band situation, roughly $1.5 \mathrm{~V}$, both electrons and holes would contribute to a conventional forward diode current. In this structure, however, electrons and holes pass the RTS, either by resonant and nonresonant tunneling or by thermionic emission across the barriers. Both carriers can therefore give rise to resonant tunneling and NDR beyond the resonance conditions. Indeed, several resonances are observed and one of them shows pronounced NDR with a peak-to-valley ratio (PVR) of 3.0 between the peak voltage $V_{p}$ at $1.73 \mathrm{~V}$ and the valley voltage $V_{v}$ at $1.83 \mathrm{~V}$. Additional resonances at $1.56,1.64$, and $1.91 \mathrm{~V}$ are more clearly observed in the differential conductance-versus-voltage plot (Fig. 1). At room temperature the PVR reduces to unity due to the larger thermionic current (not shown).

Because of the difference in effective density of states and the nearly equal doping concentration, the Fermi level in the conduction band of the $n^{+}$layer is larger than that in the valence band of the $p^{+}$layer. Furthermore, the heavy-hole concentration outnumbers the light-hole concentration by the ratio of the effective masses, i.e., approximately a factor of 12 . The transmission coefficient for heavy holes through the RTS is four orders of magnitude lower than that of the electrons, and the transmissions resonance for the heavy holes is narrower than that of the electrons. Valance-band mixing ${ }^{16-18}$ between light- and heavy-hole subbands increases the heavy-hole transmission coefficient and somewhat reduces the transmission discrepancy between electrons and heavy holes. The combined factors of carrier supply and subsequent transmission lead to a larger electron than hole tunneling current, both at onand off-resonance conditions. The observed large resonance is therefore originating from electron rcsonant tunneling in the conduction band, and the small resonances 


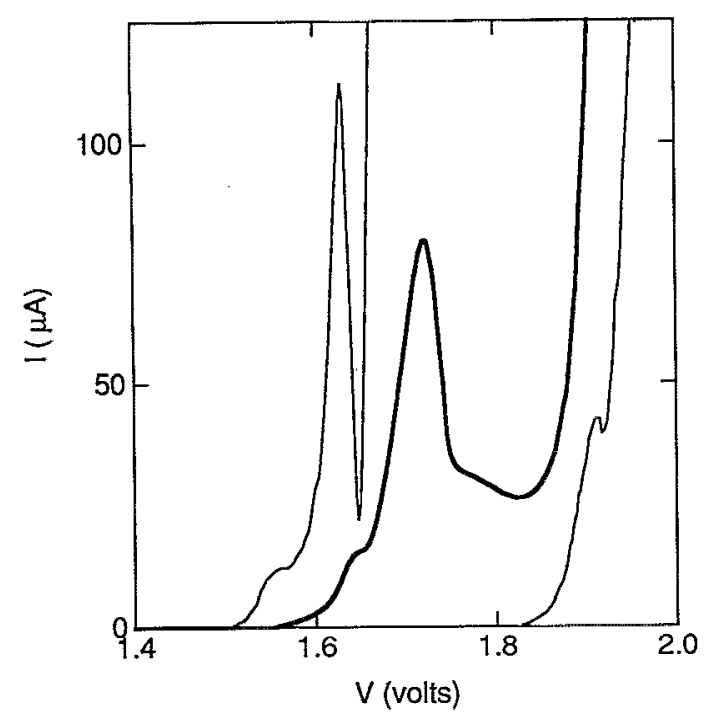

FIG. 1. Current-voltage characteristics of the bipolar RTS at $4 \mathrm{~K}$ (bold line). The differential conductance is added in arbitrary units in the region of the hole resonances (single line).

are attributed to hole resonant tunneling. The presence of hole component in the total current, by tunneling and by thermionic emission across the barriers, increases the total current at the valley of the electron resonance and leads to a reduced total current PVR at all temperatures. For this reason, the PVR of bipolar RTS will always be lower than that of unipolar RTS. This reduction in total current PVR does not directly affect the optical PVR at the electron resonance, as will be clarified from the experimental data.

The electroluminescence spectra at the peak voltage $V_{p}$ at $4 \mathrm{~K}$ are shown in Fig. 2. The quantum-well luminescence at $775 \mathrm{~nm}$ originates from recombination of electrons and holes of the first conduction-band subband and first heavy-hole valance-band subband in the quantum well, re-

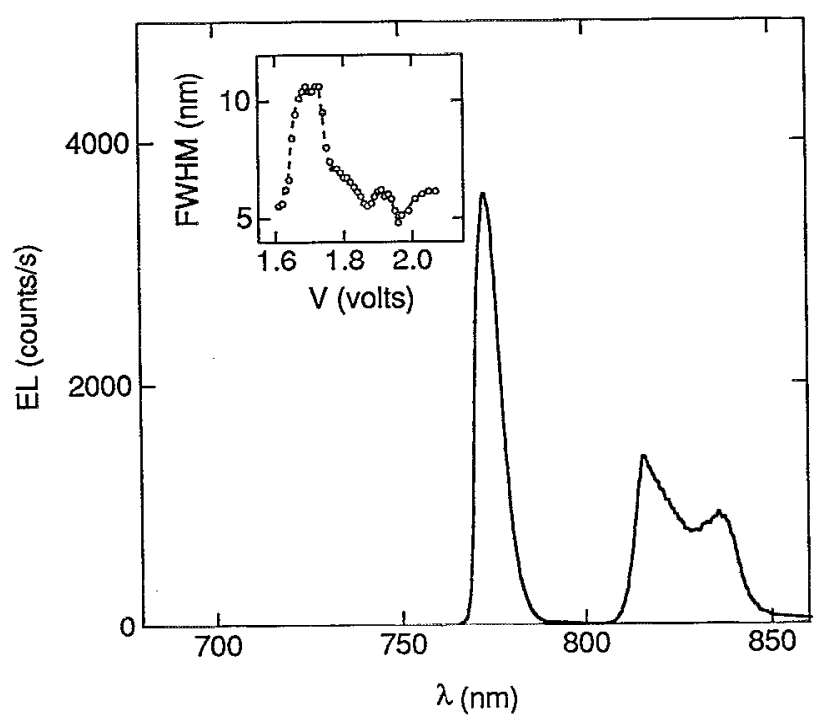

FIG. 2. Electroluminescence spectra at $4 \mathrm{~K}$ at the peak voltage of the electron resonance. The insert shows the free-carrier broadening of the quantum-well electroluminescence as a function of bias.

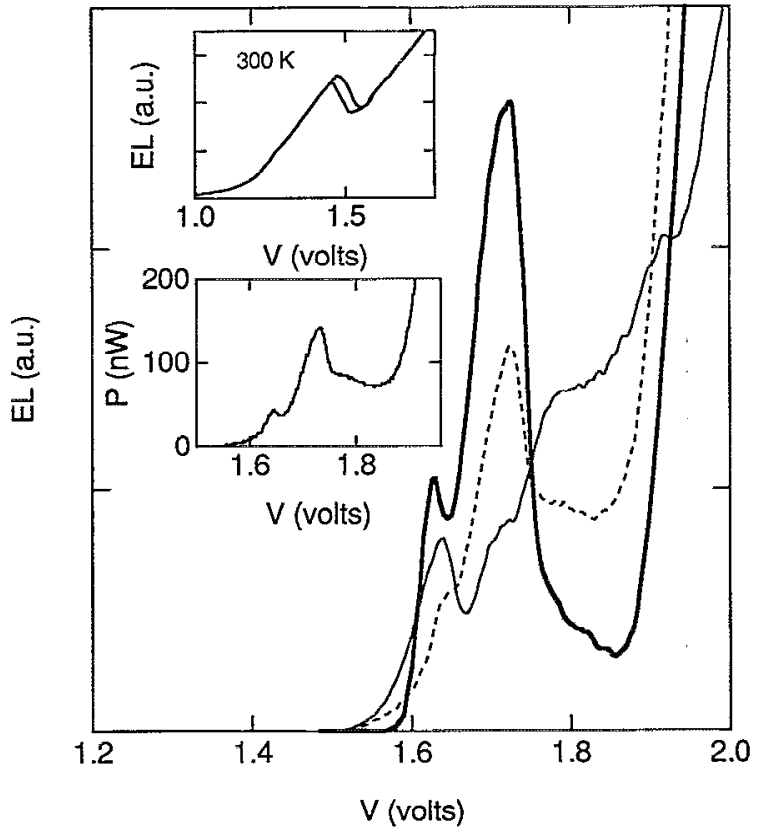

FIG. 3. Quantum-well EL (bold line), $n$-GaAs EL (single line), and $p$-GaAs EL (dashed line) vs bias at $4 \mathrm{~K}$. The bottom insert shows the total light output power of the device at $4 \mathrm{~K}$ and the top insert shows the quantum-well EL- $V$ at room tempcraturc.

spectively. In addition, $n$-GaAs and $p$-GaAs electroluminescence is observed. The insert of Fig. 2 shows the change in linewidth of the quantum-well electroluminescence due to free-carrier electron broadening at the electron resonance $(1.73 \mathrm{~V})$ and due to free-carrier hole broadening at a hole resonance $(1.91 \mathrm{~V})$.

The bias dependence of the quantum well, the $n$-GaAs, and the $p$-GaAs electroluminescence intensity (EL) at $4 \mathrm{~K}$ are shown in Fig. 3. Until flat-band conditions, the absence of both electron and hole current results in the absence of any EL. As the bias increases and reaches the onset of electron resonant tunneling at approximately $1.6 \mathrm{~V}$, the occupation of the first electron and first hole subbands increases leading to quantum-well EL. With increasing bias, the luminescence sharply increases and peaks at the voltages corresponding to the peak resonant electron and hole currents. Beyond these voltages, the luminescence decreases and reaches minima at the off-resonance conditions. As evidenced from this correlation between the $I-V$ and EL- $V$ characteristics, the quantum-well light emission reflects the electron and hole occupation of the first conduction- and valence-band subband, respectively. The strong correlation with the electron subband occupation also shows that the electrons are majority carriers in the RTS, since a breakdown in correlation can occur when the radiative recombination of the holes becomes important (in the so-called radiative limit ${ }^{14}$ ). As a result, the radiative recombination is not optimum as is also clear from the main contribution of electrons to the current-voltage characteristics. The $n$-GaAs EL (Fig. 3 ) is mostly correlated to the hole current, since it originates in the $n^{+}$contact layer and only holes are required in order to obtain electroluminescence. Similarly, the $p$-GaAs EL is more strongly cor- 
related with the electron current, since it originates in the $p^{\dagger}$ region. The observed 10:1 optical PVR of the quantum-well EL is larger than the electrical PVR at the electron resonance, since the latter is reduced by currents that do not contribute to the quantum-well EL, such as thermionic emission of electrons and holes, and a hole tunneling current. The quantum-well EL- $V$ curve at room temperature is added in the top insert of Fig. 3 and shows a reduced on-off ratio at the electron resonance. The total light power (quantum well and GaAs EL) emitted by the sample is shown in the bottom insert of Fig. 3. From the power output at the peak voltage follows a quantum efficiency of $0.3 \%$. Between the peak voltage and the valley voltage of the electron resonance, the output power reduces by a factor of 1.85 .

In conclusion, we have investigated quantum-well electroluminescence by tunneling of both electrons and holes in a bipolar RTS. A 10:1 optical PVR is observed at low temperatures that reduces to $1.45: 1$ at room temperature.

We acknowledge W. Van de Graaf for excellent support during MBE growth. J. G. acknowledges the support of the "Instituut tot Aanmoediging van het Wetenschappelijk Onderzoek in de Nijverheid en de Landbouw" (I.W.O.N.L.). E. G. is a fellow of the Belgian National Fund for Scientific Research (NFWO).

Note added in Proof: L. Eaves presented resonant tunneling light-emitting diodes at the 21st European SolidState Device Research Conference (ESSDERC91), Lausanne, Switzerland.

${ }^{1}$ E. T. Yu, M. K. Jackson, and T. C. McGill, Appl. Phys. Lett. 55, 744 (1989).
${ }^{2}$ V. J. Goldman, D. C. Tsui, and J. E. Cunningham, Phys. Rev. B 35, 9387 (1987).

${ }^{3}$ F. Capasso, S. Sen, F. Beltram, L. M. Lunardi, A. S. Vengurlekar, P. R. Smith, N. J. Shah, R. J. Malik, and A. Y. Cho, IEEE Trans. Electron Devices ED-36, 2065 (1989), and references therein.

${ }^{4}$ R. C. Potter, A. A. Lakhani, D. Beyea, E. Hempling, and A. Fathimulla, Appl. Phys. Lett. 52, 2163 (1988).

${ }^{5}$ I. Mehdi, G. I. Haddad, and R. K. Mains, Superlattices and Microstructures 5, 443 (1989).

${ }^{t}$ P. England, J. E. Golub, L.T. Florez, and J. P. Harbison, Appl. Phys. Lett. 58. 887 (1991).

${ }^{7}$ J. F. Young, B. M. Wood, G. C. Aers, R. L. S. Devine, H. C. Liu, M. Buchanan, A. J. SpringThorpe, and P. Mandeville, Phys. Rev. Lett. 20, 2085 (1988).

${ }^{8}$ J. F. Young, B. M. Wood, G. C. Aers, R. L. S. Devine, H. C. Liu, M. Buchanan, A. J. SpringThorpe, and P. Mandeville, Superlattices and Microstructures 5, 411 (1989).

${ }^{9}$ N. Vodjdani, F. Chevoir, D. Thomas, D. Cote, P. Bois, E. Costard, and S. Delaitre, Appl. Phys. Lett. 55, 1528 (1989).

${ }^{10}$ L. Eaves, M. L. Leadbeater, D. G. Hayes, E. S. Alves, F. W. Sheard, G. A. Toombs, P. E. Simmonds, M S. Skolnic, M. Henini, and O. H. Huges, Solid-State Electron. 32, 1101 (1989).

${ }^{11}$ M. S. Skolnick, D. G. Hayes, P. E. Simmonds, A. W. Higgs, G. W. Smith, H. J. Hutchinson, C. R. Whitehouse, L. Eaves, M. Henini, O. H. Hughes, M. L. Leadbeater, and D P. Halliday, Phys. Rev. B 41, 10754 (1990).

${ }^{12}$ C. Van Hoof, E. Goovaerts, and G. Borghs, Proc. SPIE 1362, 291 (1991).

${ }^{13}$ N. Vodjdani, D. Cote, D. Thomas, B. Sermage, P. Bois, E. Costard, and J. Nagle, Appl. Phys. Lett. 56, 33 (1990).

${ }^{14}$ M. S. Skolnick, P. E. Simmonds, D. G. Hayes, A. W. Higgs, G. W. Smith, A. D. Pitt, C. R. Whitehouse, H. J. Hutchinson, C. R. H. White, L. Eaves, M. Henini, and O. H. Hughes, Phys. Rev. B 42, 3069 (1990).

${ }^{15}$ C. R. H. White, M. S. Skolnick, L. Eaves, and M. L. Leadbeater, Appl. Phys. Lett. 11, 1164 (1991).

${ }^{16}$ R. K. Hayden, D. K. Maude, L. Eaves, E. C. Valadares, M. Henini, F. W. Sheard, O. Hughes, J. C. Portal, and L. Cury, Phys. Rev. Lett. 66, 1749 (1991).

${ }^{17}$ C. Y. Chao and S. L. Chuang, Phys. Rev. B 43, 7027 (1991).

${ }^{18}$ R. Wessel and M. Altarelli, Phys. Rev. B 39, 12802 (1989). 